\title{
Kybernetika
}

\section{Ján Plavka}

Computing the greatest $\mathbf{X}$-eigenvector of a matrix in max-min algebra

Kybernetika, Vol. 52 (2016), No. 1, 1-14

Persistent URL: http://dml.cz/dmlcz/144857

\section{Terms of use:}

(C) Institute of Information Theory and Automation AS CR, 2016

Institute of Mathematics of the Czech Academy of Sciences provides access to digitized documents strictly for personal use. Each copy of any part of this document must contain these Terms of use.

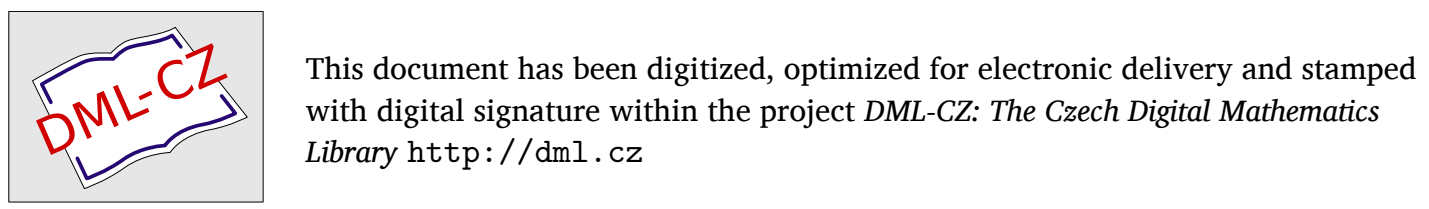




\title{
COMPUTING THE GREATEST X-EIGENVECTOR OF A MATRIX IN MAX-MIN ALGEBRA
}

\author{
JÁN PlavkA
}

A vector $x$ is said to be an eigenvector of a square max-min matrix $A$ if $A \otimes x=x$. An eigenvector $x$ of $A$ is called the greatest $\boldsymbol{X}$-eigenvector of $A$ if $x \in \boldsymbol{X}=\{x ; \underline{x} \leq x \leq \bar{x}\}$ and $y \leq x$ for each eigenvector $y \in \boldsymbol{X}$. A max-min matrix $A$ is called strongly $\boldsymbol{X}$-robust if the orbit $x, A \otimes x, A^{2} \otimes x, \ldots$ reaches the greatest $\boldsymbol{X}$-eigenvector with any starting vector of $\boldsymbol{X}$. We suggest an $O\left(n^{3}\right)$ algorithm for computing the greatest $\boldsymbol{X}$-eigenvector of $A$ and study the strong $\boldsymbol{X}$-robustness. The necessary and sufficient conditions for strong $\boldsymbol{X}$-robustness are introduced and an efficient algorithm for verifying these conditions is described.

Keywords: eigenvector, interval vector, max-min matrix

Classification: 08A72, 90B35, 90C47

\section{INTRODUCTION}

Matrices in $\max -\min$ algebra (the addition and the multiplication are formally replaced by operations of maximum and minimum) can be used in a range of practical problems related to scheduling, optimization, modeling of fuzzy discrete dynamic systems, graph theory, knowledge engineering, cluster analysis, fuzzy systems and also related to describing diagnosis of technical devices [21] or medical diagnosis [18.

The research of max-min algebra can be motivated by adapting max-plus multiprocessor interaction systems [1. In these systems we have $n$ processors which work in stages, and in the algebraic model of their interactive work, entry $x_{i}(k)$ of a vector $x(k)$, represents the state of processor $i$ after some stage $k$, and the entry $a_{i j}$ of a matrix $A$ encodes the influence of the work of processor $j$ in the previous stage on the work of processor $i$ in the current stage. For simplicity, the system is assumed to be homogeneous, so that $A$ does not change from stage to stage. Summing up all the influence effects multiplied by the results of previous stages, we have $x_{i}(k+1)=$ $\bigoplus_{j} a_{i j} \otimes x_{j}(k)$. The summation is often interpreted as waiting till all works of the system are finished and all the necessary influence constraints are satisfied.

Thus the orbit $x, A \otimes x, \ldots A^{k} \otimes x$, where $A^{k}=A \otimes \ldots \otimes A$, represents the evolution of such a system. Regarding the orbits, one wishes to know the set of starting vectors from which a steady state of multi-processor interaction systems (an eigenvector of $A$;

DOI: $10.14736 / \mathrm{kyb}-2016-1-0001$ 
$A \otimes x=x$ ) can be achieved. The set of starting vectors from which a system reaches an eigenvector (the greatest eigenvector) of $A$ after a finite number of stages, in general, contains the set of all eigenvectors, but it can be an interval vector $\boldsymbol{X}=[\underline{x}, \bar{x}]:=\{x ; \underline{x} \leq$ $x \leq \bar{x}\}$ and also as big as the whole space.

In the present paper, we consider a generalized version of the problem to compute the greatest eigenvector of $A$ (see [2]) belonging to an interval vector $\boldsymbol{X}$ (called the greatest $\boldsymbol{X}$-eigenvector of $A$ ). In Theorem 5.8 , which is the main result of the paper, we show that under a certain natural condition, the greatest $\boldsymbol{X}$-eigenvector of $A$ can be computed by an $O\left(n^{3}\right)$ algorithm.

The next section will be occupied by some definitions and notation of the max-min algebra, leading to the discussion of the greatest $\boldsymbol{X}$-eigenvector of the matrix $A$ and strong $\boldsymbol{X}$-robustness of $A$. Section 5 is devoted to the main result characterizing strong $\boldsymbol{X}$-robust matrices with an orbit of $A$.

Let us conclude with a brief overview of the works on max-min algebra to which this paper is related. The paper [2] deals with the problem of computing the greatest eigenvector of a given max-min matrix. The concepts of robustness (an eigenvector of $A$ is reached with any starting vector) and strong robustness (the greatest eigenvector of $A$ is reached with any starting vector) in max-min algebra were introduced and studied in [14] and [16]. Following that work, some equivalent conditions and efficient algorithms were presented in [10, 11, 16]. In particular, see [16] for some polynomial procedures checking the weak robustness (an eigenvector is reached only if a starting vector is an eigenvector of $A$ ) in max-min algebra.

\section{PRELIMINARIES}

Let $(\mathbb{B}, \leq)$ be a bounded linearly ordered set with the least element in $\mathbb{B}$ denoted by $O$ and the greatest one by $I$. The set of naturals (naturals with zero) is denoted by $\mathbb{N}\left(\mathbb{N}_{0}\right)$. For given naturals $n, m \in \mathbb{N}$, we use the notations $N$ and $M$ for the set of all smaller or equal natural numbers, i.e., $N=\{1,2, \ldots, n\}$ and $M=\{1,2, \ldots, m\}$, respectively. The set of $n \times m$ matrices over $\mathbb{B}$ is denoted by $\mathbb{B}(n, m)$, specially the set of $n \times 1$ vectors over $\mathbb{B}$ is denoted by $\mathbb{B}(n)$.

The max-min algebra is a triple $(\mathbb{B}, \oplus, \otimes)$, where $a \oplus b=\max (a, b)$ and $a \otimes b=$ $\min (a, b)$.

The operations $\oplus, \otimes$ are extended to the matrix-vector algebra over $\mathbb{B}$ by the direct analogy to the conventional linear algebra. If each entry of a matrix $A \in \mathbb{B}(n, n)$ (a vector $x \in \mathbb{B}(n))$ is equal to $O$ we shall denote it as $A=O(x=O)$.

The greatest common divisor and the least common multiple of a set $S \subseteq \mathbb{N}$ is denoted by $\operatorname{gcd} S$ and $\operatorname{lcm} S$, respectively.

For $A \in \mathbb{B}(n, n), C \in \mathbb{B}(n, n)$ we write $A \leq C(A<C)$ if $a_{i j} \leq c_{i j}\left(a_{i j}<c_{i j}\right)$ holds true for all $i, j \in N$. Similarly, for $x=\left(x_{1}, \ldots, x_{n}\right)^{T} \in \mathbb{B}(n)$ and $y=\left(y_{1}, \ldots, y_{n}\right)^{T} \in$ $\mathbb{B}(n)$ we write $x \leq y(x<y)$ if $x_{i} \leq y_{i}\left(x_{i}<y_{i}\right)$ for each $i \in N$.

The $r$ th power of a matrix $A$ is denoted by $A^{r}$ with elements $a_{i j}^{r}$.

By digraph we understand a pair $G=\left(V_{G}, E_{G}\right)$, where $V_{G}$ is a non-empty finite set, called the node set, and $E_{G} \subseteq V_{G} \times V_{G}$, called the arc set. A digraph $G^{\prime}$ is a subdigraph of $G$, if $V_{G^{\prime}} \subseteq V_{G}$ and $E_{G^{\prime}} \subseteq E_{G}$. A path in $G$ is the sequence of nodes $p=\left(v_{0}, v_{1}, \ldots, v_{l}\right)$ such that $\left(v_{k-1}, v_{k}\right) \in E_{G}$ for all $k=1,2, \ldots, l$ (denoted as $\left(v_{0}, v_{l}\right)$-path). The number 
$l(p) \geq 0$ is called the length of $p$. If $v_{0}=v_{l}$, then $p$ is called a cycle. A cycle is elementary if all nodes except the terminal node are distinct. A digraph is called strongly connected if any two distinct nodes of $G$ are contained in a common cycle.

By a strongly connected component $\mathcal{K}$ of $G=(N, E)$ we mean a subdigraph $\mathcal{K}$ generated by a non-empty subset $K \subseteq N$ such that any two distinct nodes $i, j \in K$ are contained in a common cycle and $K$ is the maximal subset with this property. A strongly connected component $\mathcal{K}$ of a digraph is called non-trivial, if there is a cycle of positive length in $\mathcal{K}$. For any non-trivial strongly connected component $\mathcal{K}$ the period of $\mathcal{K}$ is defined as per $\mathcal{K}=\operatorname{gcd}\{l(c) ; c$ is a cycle in $\mathcal{K}, l(c)>0\}$. If $\mathcal{K}$ is trivial, then per $\mathcal{K}=1$.

There is a well-known connection between the entries in powers of matrices and paths in associated digraphs: the $(i, j)$ th entry $a_{i j}^{k}$ in $A^{k}$ is equal to the maximum of weights of paths from $\mathcal{P}_{i j}^{k}$, where $\mathcal{P}_{i j}^{k}$ is the set of all paths of length $k$ beginning at node $i$ and ending at node $j$. If $\mathcal{P}_{i j}$ denotes the set of all paths from $i$ to $j$, then $a_{i j}^{*}=\max \left\{a_{i j}^{k} ; k=1,2, \ldots\right\}$ is the maximum weight of a path from $\mathcal{P}_{i j}$ and $a_{j j}^{*}$ is the maximum weight of a cycle containing node $j$.

For a given matrix $A \in \mathbb{B}(n, n)$, the number $\lambda \in \mathbb{B}$ and the $n$-tuple $x \in \mathbb{B}(n)$ are the so-called eigenvalue of $A$ and eigenvector of $A$, respectively, if $A \otimes x=\lambda \otimes x$.

The eigenspace $V(A, \lambda)$ is defined as the set of all eigenvectors of $A$ with associated eigenvalue $\lambda$, i.e., $V(A, \lambda)=\{x \in B(n) ; A \otimes x=\lambda \otimes x\}$.

In case $\lambda=I$ let us denote $V(A, I)$ by abbreviation $V(A)$.

Define the greatest eigenvector $x^{*}(A)$ corresponding to a matrix $A$ and the eigenvalue $I$ as $x^{*}(A)=\bigoplus_{x \in V(A)} x$.

In 2 it was stated that the greatest eigenvector $x^{*}(A)$ exists for every matrix $A$, its entries are given by the formula $x_{i}^{*}(A)=\bigoplus_{j} a_{i j}^{*} \otimes a_{j j}^{*}$ and $x^{*}(A)$ can be computed by an $O\left(n^{3}\right)$ iterative procedure.

For every matrix $A \in \mathbb{B}(n, n)$ denote

$$
c_{i}(A)=\bigoplus_{j \in N} a_{i j}, \quad c(A)=\bigotimes_{i \in N} c_{i}(A), \quad c^{*}(A)=(c(A), \ldots, c(A))^{T} \in \mathbb{B}(n) .
$$

\section{ORBIT PERIODICITY}

The notions of an orbit of $A$ generated by $x$ and known properties of the orbit periodicity are introduced in this section.

Definition 3.1. For any $A \in \mathbb{B}(n, n)$ and $x \in \mathbb{B}(n)$ the orbit of $A$ generated by $x$ is the vector sequence $\mathcal{O}(A, x)=\left(x(r) ; r \in \mathbb{N}_{0}\right)$ whose initial vector is $x(0)=x$ and successive members are defined by the formula $x(r+1)=A \otimes x(r)$.

Definition 3.2. The sequence $S=(S(r) ; r \in \mathbb{N})$ is ultimately periodic if there is a natural number $p$ such that the following holds for some natural number $R: S(k+p)=$ $S(k)$ for all $k \geq R$. The smallest natural number $p$ with the above property is called the period of $S$, denoted by $\operatorname{per}(S)$. 
Both operations in max-min algebra are idempotent, hence no new numbers are created in the process of generating an orbit. Therefore any orbit in max-min algebra contains only a finite number of different vectors. Thus an orbit is always ultimately periodic. The same holds true for the power sequence $\left(A^{k} ; k \in \mathbb{N}\right)$. Hence a power sequence and an orbit $\mathcal{O}(A, x)$ are always ultimately periodic sequences. Their periods will be called the period of $A$ and the orbit period of $\mathcal{O}(A, x)$, in notation $\operatorname{per}(A), \operatorname{per}(A, x)$.

Theorem 3.3. (Gavalec [5]) Let $A \in \mathbb{B}(n, n)$ and $x \in \mathbb{B}(n)$.

Then $\operatorname{per}(A)=\operatorname{lcm}_{x \in B(n)} \operatorname{per}(A, x)$.

Note that an $O\left(n^{3}\right)$ algorithm for finding $\operatorname{per}(A)$ is presented in [5].

A matrix (vector) is called binary if $a_{i j} \in\{O, I\}\left(x_{j} \in\{O, I\}\right)$ for each $i, j \in N$.

Definition 3.4. Let $A \in \mathbb{B}(n, n)$ be a binary matrix and $x \in \mathbb{B}(n)$ be a binary vector. Then by $G(A)=\left(V_{G(A)}, E_{G(A)}\right)$ we understand the digraph with $V_{G(A)}=N, E_{G(A)}=$ $\left\{(i, j) ; a_{i j}=I\right\}$ and by $G(A, x)$ we understand the corresponding node-weighted digraph obtained from $G(A)$ by appending weight $x_{i}$ to each node $i$ (denoted by $i^{O}$ if $x_{i}=O$ and $i^{I}$ if $\left.x_{i}=I\right)$.

A path in $G(A, x)$ is called an orbit path if the weight of its terminal node is $I$.

Definition 3.5. For $A \in \mathbb{B}(n, n), x \in \mathbb{B}(n)$ and $h \in \mathbb{B}$, the threshold matrix $A_{(h)}$ and threshold vector $x_{(h)}$ corresponding to the threshold $h$ is a binary matrix of the same type as $A$ and a binary vector of the same type as $x$, defined as follows:

$$
\left(a_{(h)}\right)_{i j}=\left\{\begin{array}{ll}
I, & \text { if } a_{i j} \geq h, \\
O, & \text { otherwise },
\end{array} \text { and } \quad\left(x_{(h)}\right)_{i}= \begin{cases}I, & \text { if } x_{i} \geq h \\
O, & \text { otherwise }\end{cases}\right.
$$

respectively. The associated digraphs $G\left(A_{(h)}\right)$ and $G\left(A_{(h)}, x_{(h)}\right)$ will be called the threshold digraphs corresponding to the threshold $h$.

Definition 3.6. Let $k \in N$ be a node of $G\left(A_{(h)}, x_{(h)}\right)$. $k$ is called removable if there is a node $j \in N$ such that $(j, k)$-path is an orbit path in $G\left(A_{(h)}, x_{(h)}\right)$ with $\left(x_{(h)}\right)_{j}=O$. If $\left(x_{(h)}\right)_{k}=O$ then $k$ is removable by the definition.

Let $G\left(A_{(h)}, x_{(h)}\right)$ be the threshold digraph corresponding to the threshold $h$. Denote $\tilde{G}\left(A_{(h)}, x_{(h)}\right)$ the digraph which arose from $G\left(A_{(h)}, x_{(h)}\right)$ by deleting all removable nodes.

Similarly as in [2], a node $k \in N$ is called precyclic in $\tilde{G}\left(A_{(h)}, x_{(h)}\right)$ if there is a path $p$ that is finished by a cycle, i.e., $p=\left(k, v_{1}, \ldots, v_{t}, \ldots, v_{t+s}, v_{t}\right)$. If $k$ is lying on a cycle then $k$ is precyclic by the definition.

A characterization of $\tilde{G}\left(A_{(h)}, x_{(h)}\right)$ and a description of precyclic node is necessary for computing the greatest eigenvector belonging to an interval vector $\boldsymbol{X}$. This extension of the theory presented in [2] is done in the next section. 


\section{INTERVAL VECTORS}

In this section we shall deal with properties of the greatest eigenvector belonging to an interval vector.

Similarly to [3, 4, 6, 17, 8, 9, 11, 12, 13, 15, 17, we define an interval vector $\boldsymbol{X}$.

Definition 4.1. Let $\underline{x}, \bar{x} \in \mathbb{B}(n), \underline{x} \leq \bar{x}$. An interval matrix $\boldsymbol{X}$ with bounds $\underline{x}$ and $\bar{x}$ is defined as follows $\boldsymbol{X}=[\underline{x}, \bar{x}]=\{x \in \mathbb{B}(n) ; \underline{x} \leq x \leq \bar{x}\}$.

For a given $A \in \mathbb{B}(n, n)$ and $\boldsymbol{X} \subseteq \mathbb{B}(n)$ define the greatest $\boldsymbol{X}$-eigenvector $x^{*}(A, \boldsymbol{X})$ corresponding to a matrix $A$ and an interval vector $\boldsymbol{X}$ as $x^{*}(A, \boldsymbol{X})=\bigoplus_{x \in V(A) \cap \boldsymbol{X}^{x}}$. As it has been written, if $\boldsymbol{X}=\mathbb{B}(n)$ then $x^{*}(A, \boldsymbol{X})$ exists for every matrix $A$ (see [2, 20]), this is in a contrast with the case that $\boldsymbol{X} \subset \mathbb{B}(n)$ for which $x^{*}(A, \boldsymbol{X})$ exists if only if $V(A) \cap \boldsymbol{X} \neq \emptyset$.

Now, we define an auxiliary eigenvector $x^{\oplus}(A, \boldsymbol{X})$ of $A$ belonging to $\boldsymbol{X}$ which allows us to use properties of digraphs and to characterize the structure of the greatest $\boldsymbol{X}$ eigenvector $x^{*}(A, \boldsymbol{X})$ corresponding to a matrix $A$ and an interval vector $\boldsymbol{X}$.

Definition 4.2. For a given $A \in \mathbb{B}(n, n)$ and $\boldsymbol{X} \subseteq \mathbb{B}(n)$ define a vector $x^{\oplus}(A, \boldsymbol{X})=$ $\left(x_{1}^{\oplus}(A, \boldsymbol{X}), \ldots, x_{n}^{\oplus}(A, \boldsymbol{X})\right)^{T}$ as follows

$$
x_{k}^{\oplus}(A, \boldsymbol{X})=\max \left\{h \in\left[\underline{x}_{k}, \bar{x}_{k}\right] ; k \text { is precyclic in } \tilde{G}\left(A_{(h)}, \bar{x}_{(h)}\right)\right\} .
$$

Notice that if there is $k \in N$ such that $\left\{h \in\left[\underline{x}_{k}, \bar{x}_{k}\right] ; k\right.$ is precyclic in $\left.\tilde{G}\left(A_{(h)}, \bar{x}_{(h)}\right)\right\}=\emptyset$ then $x_{k}^{\oplus}(A, \boldsymbol{X})$ does not exist.

Example 4.3. Let $A$ and $\boldsymbol{X}=[\underline{x}, \bar{x}]$ have the forms

$$
A=\left(\begin{array}{llll}
1 & 3 & 8 & 6 \\
0 & 4 & 6 & 2 \\
5 & 4 & 6 & 1 \\
9 & 4 & 3 & 2
\end{array}\right), \quad \underline{x}=\left(\begin{array}{l}
4 \\
4 \\
3 \\
4
\end{array}\right), \quad \bar{x}=\left(\begin{array}{l}
8 \\
5 \\
6 \\
9
\end{array}\right)
$$

Then needed digraphs are displayed in Figures 1-5. 


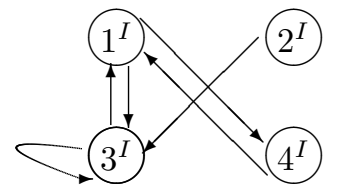

Fig. 1. $G\left(A_{(5)}, x_{(5)}\right)=\tilde{G}\left(A_{(5)}, x_{(5)}\right)$.

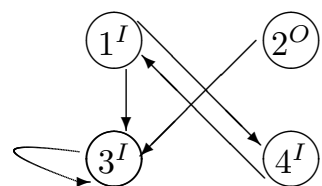

Fig. 2. $G\left(A_{(6)}, x_{(6)}\right)$.

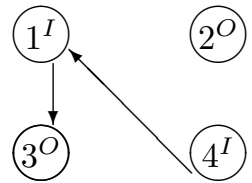

Fig. 4. $G\left(A_{(7)}, x_{(7)}\right)$.

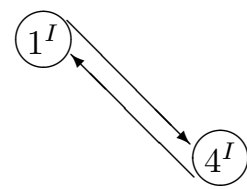

Fig. 3. $\tilde{G}\left(A_{(6)}, x_{(6)}\right)$.

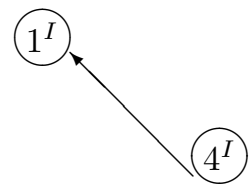

Fig. 5. $\tilde{G}\left(A_{(7)}, x_{(7)}\right)$.

By the definition of $x_{k}^{\oplus}(A, \boldsymbol{X})$ we get that node 2 and node 3 are precyclic in $\tilde{G}\left(A_{(5)}, x_{(5)}\right)$ and nodes 1, 4 are precyclic in $\tilde{G}\left(A_{(6)}, x_{(6)}\right)$. The graph $\tilde{G}\left(A_{(7)}, x_{(7)}\right)$ (similarly as $\tilde{G}\left(A_{(8)}, x_{(8)}\right)$ and $\left.\tilde{G}\left(A_{(9)}, x_{(9)}\right)\right)$ is acyclic and hence we get that $x^{\oplus}(A, \boldsymbol{X})=(6,5,5,6)^{T}$.

From now we shall suppose that $x_{k}^{\oplus}(A, \boldsymbol{X})$ exists. Then from the last definition the next lemma straightly follows.

Lemma 4.4. Let $A$ and $\boldsymbol{X}$ be given. Then $\underline{x} \leq x^{\oplus}(A, \boldsymbol{X}) \leq \bar{x}$.

Theorem 4.5. Let $A$ and $\boldsymbol{X}$ be given. Then $x^{\oplus}(A, \boldsymbol{X}) \in V(A)$.

Proof. Consider an arbitrary but fixed $k \in N$ and suppose that $k$ is precyclic in $\tilde{G}\left(A_{(h)}, \bar{x}_{(h)}\right)$ for $h=x_{k}^{\oplus}(A, \boldsymbol{X})$. Then there is a path $p=\left(k, v_{1}, \ldots, v_{t}, \ldots, v_{t+s}, v_{t}\right)$ such that $\left(v_{t}, \ldots, v_{t+s}, v_{t}\right)$ is a cycle and each node $v_{j} \in p$ is precyclic in $\tilde{G}\left(A_{(h)}, \bar{x}_{(h)}\right)$. Moreover $x_{v_{1}}^{\oplus}(A, \boldsymbol{X}) \geq x_{k}^{\oplus}(A, \boldsymbol{X})=h$ and $a_{k v_{1}} \geq h$. Hence we get

$$
\left(A \otimes x^{\oplus}(A, \boldsymbol{X})\right)_{k}=\bigoplus_{j \in N} a_{k j} \otimes x_{j}^{\oplus}(A, \boldsymbol{X}) \geq a_{k v_{1}} \otimes x_{v_{1}}^{\oplus}(A, \boldsymbol{X}) \geq x_{k}^{\oplus}(A, \boldsymbol{X}) .
$$


To prove the reverse inequality suppose for a contrary that there is some index $\ell$ such that $a_{k \ell} \otimes x_{\ell}^{\oplus}(A, \boldsymbol{X})>x_{k}^{\oplus}(A, \boldsymbol{X})$. Node $\ell$ is precyclic in $\tilde{G}\left(A_{(h)}, \bar{x}_{(h)}\right)$ for $h=x_{\ell}^{\oplus}(A, \boldsymbol{X})$, i.e., there is a path $p^{\prime}=\left(\ell, u_{1}, \ldots, u_{t}, \ldots, u_{t+s}, u_{t}\right)$. Then we can construct a new path $\tilde{p}^{\prime}=\left(k, \ell, u_{1}, \ldots, u_{t}, \ldots, u_{t+s}, u_{t}\right)$ which guarantees that $k$ is precyclic in $\tilde{G}\left(A_{\left(h^{\prime}\right)}, \bar{x}_{\left(h^{\prime}\right)}\right)$ for $h^{\prime}=a_{k \ell} \otimes x_{\ell}^{\oplus}(A, \boldsymbol{X})$. This is a contradiction with the definition of $x_{k}^{\oplus}(A, \boldsymbol{X})$.

Theorem 4.6. Let $A$ and $\boldsymbol{X}$ be given. Then $(\forall x \in V(A) \cap \boldsymbol{X})\left[x \leq x^{\oplus}(A, \boldsymbol{X})\right]$.

Pr o of. Suppose that $A, \boldsymbol{X}$ and $x \in V(A) \cap \boldsymbol{X}$ are given. Then $(A \otimes x)_{k}=x_{k}$ implies that there is $j_{1} \in N$ such that $a_{k j_{1}} \otimes x_{j_{1}}=x_{k}$ and $a_{k j_{1}} \geq x_{k} \wedge x_{j_{1}} \geq x_{k}$. For row index $j_{1}$ there is $j_{2}$ such that $a_{j_{1} j_{2}} \otimes x_{j_{2}}=x_{j_{1}}$ and $a_{j_{1} j_{2}} \geq x_{j_{1}} \wedge x_{j_{2}} \geq x_{j_{1}}$. By repeating the above process at most $n$ times we obtain a path $p=\left(k, j_{1}, \ldots, j_{s}, j_{s+1}, \ldots, j_{s}\right)$, i.e., $k$ is precyclic in $\tilde{G}\left(A_{\left(x_{k}\right)}, \bar{x}_{\left(x_{k}\right)}\right)$. Notice that no node of $p$ can be removable (if $j_{l}$ is removable then there is a path $p^{\prime}=\left(i_{1}, \ldots, i_{s}=j_{l}\right)$ in $G\left(A_{\left(x_{k}\right)}, \bar{x}_{\left(x_{k}\right)}\right)$ with $i_{v}^{O}$ and $i_{v+1}^{I}$ and hence inequality $a_{i_{s} i_{s+1}} \otimes x_{i_{s+1}}>x_{i_{s}}$ contradicts the assumption that $\left.A \otimes x=x\right)$. The assertion follows from the fact that $x_{k}^{\oplus}(A, \boldsymbol{X})$ is equal to the greatest $h$ for which $k$ is precyclic in $\tilde{G}\left(A_{(h)}, \bar{x}_{(h)}\right)$.

In the last three assertions we have showed that $x^{\oplus}(A, \boldsymbol{X})$ is an eigenvector belonging to $\boldsymbol{X}$ and fulfilling a maximality condition $\left((\forall x \in V(A) \cap \boldsymbol{X})\left[x \leq x^{\oplus}(A, \boldsymbol{X})\right]\right.$. This is a reason to formulate the next corollary.

Corollary 4.7. If $V(A) \cap \boldsymbol{X} \neq \emptyset$ then $x^{*}(A, \boldsymbol{X})=x^{\oplus}(A, \boldsymbol{X})$.

\section{PROCEDURE FOR COMPUTING THE GREATEST X-EIGENVECTOR}

The task to compute $x^{\oplus}(A, \boldsymbol{X})$ more effectively is considered in this section.

Definition 5.1. Let $A$ and $\boldsymbol{X}$ be given. We say that $\boldsymbol{X}$ is invariant under $A$ if $x \in \boldsymbol{X}$ implies $A \otimes x \in \boldsymbol{X}$.

As $A$ is order-preserving, the invariance of $\boldsymbol{X}$ under $A$ admits the following simple characterization.

Lemma 5.2. $\boldsymbol{X}$ is invariant under $A$ if and only if $\underline{x} \leq A \otimes \underline{x} \wedge A \otimes \bar{x} \leq \bar{x}$.

Suppose that $\boldsymbol{X}$ is invariant under $A$ and $\mathcal{O}(A, \bar{x})=\left(\bar{x}(r) ; r \in \mathbb{N}_{0}\right)$ and $\mathcal{O}(A, \underline{x})=$ $\left(\underline{x}(r) ; r \in \mathbb{N}_{0}\right)$ are orbits of $A$ generated by $\bar{x}$ and $\underline{x}$, respectively. Then for each $k \in \mathbb{N}_{0}$ we have the following:

$$
\begin{aligned}
& \bar{x}(k+1)=A^{k+1} \otimes \bar{x}=A^{k} \otimes(A \otimes \bar{x}) \leq A^{k} \otimes \bar{x}=\bar{x}(k) . \\
& \underline{x}(k+1)=A^{k+1} \otimes \underline{x}=A^{k} \otimes(A \otimes \underline{x}) \geq A^{k} \otimes \underline{x}=\underline{x}(k) .
\end{aligned}
$$


Lemma 5.3. Let $\boldsymbol{X}$ be invariant under $A$. Then

$$
\begin{aligned}
& \left(\forall k \in \mathbb{N}_{0}\right)[\bar{x}(n)=\bar{x}(n+k)], \\
& \left(\forall k \in \mathbb{N}_{0}\right)[\underline{x}(n)=\underline{x}(n+k)] .
\end{aligned}
$$

Proof. According to (2) it is sufficient to prove that $\bar{x}(n) \leq \bar{x}(n+1)$. For the sake of a contradiction assume that $\bar{x}(n) \not \leq \bar{x}(n+1)$, i.e., there is $i \in N$ such that $\bar{x}_{i}(n)>\bar{x}_{i}(n+1)$, i.e., $\left(A^{n} \otimes \bar{x}\right)_{i}>\left(A^{n+1} \otimes \bar{x}\right)_{i}$. Then there is $s \in N$ such that for each $k \in N$ the following inequality holds true $a_{i s}^{n} \otimes \bar{x}_{s}>a_{i k}^{n+1} \otimes \bar{x}_{k}$, or equivalently, there is a path $p \in \mathcal{P}_{i s}^{n}$ such that next formula

$$
w(p) \otimes \bar{x}_{s}=a_{i s}^{n} \otimes \bar{x}_{s}>a_{i k}^{n+1} \otimes \bar{x}_{k} \geq w(\tilde{p}) \otimes \bar{x}_{k}
$$

holds for each $k \in N$ and each $\tilde{p} \in \mathcal{P}_{i k}^{n+1}$. Since $l(p)=n$ then there is at least one repeated node, i.e., $p=p^{\prime} \cup c$ and $l(c) \geq 1$. Now, we shall consider a path $p^{\prime \prime}$ such that $p^{\prime \prime}=p^{\prime} \cup c \cup c$. Let $l\left(p^{\prime \prime}\right)=v$. Then $v \geq n+1$ and we get

$$
\bar{x}_{i}(v) \geq a_{i s}^{v} \otimes \bar{x}_{s} \geq w\left(p^{\prime \prime}\right) \otimes \bar{x}_{s}=w(p) \otimes \bar{x}_{s}=a_{i s}^{n} \otimes \bar{x}_{s}>a_{i k}^{n+1} \otimes \bar{x}_{k}=\bar{x}_{i}(n+1) .
$$

This is a contradiction with (2) and (4) follows.

To prove (5) it is enough (by (4)) to show that $\underline{x}(n) \geq \underline{x}(n+1)$. For the sake of a contradiction assume that $\underline{x}(n) \nsupseteq \underline{x}(n+1)$, i.e., there is $i \in N$ such that $\underline{x}_{i}(n)<\underline{x}_{i}(n+1)$ or equivalently $\left(A^{n} \otimes \underline{x}\right)_{i}<\left(A^{n+1} \otimes \underline{x}\right)_{i}$. Then there is $k \in N$ such that for each $s \in N$ the following inequality holds true $a_{i s}^{n} \otimes \underline{x}_{s}<a_{i k}^{n+1} \otimes \underline{x}_{k}$, or again equivalently, there is a path $p \in \mathcal{P}_{i k}^{n+1}$ such that next formula

$$
w\left(p^{\prime}\right) \otimes \underline{x}_{s} \leq a_{i s}^{n} \otimes \underline{x}_{s}<a_{i k}^{n+1} \otimes \underline{x}_{k}=w(p) \otimes \underline{x}_{k}
$$

holds for each $s \in N$ and each $p^{\prime} \in \mathcal{P}_{i s}^{n}$. Since $l(p)=n+1$ then there is at least one repeated node, i.e., $p=\tilde{p} \cup c$, where $\tilde{p} \in \mathcal{P}_{i k}^{l(\tilde{p})}$ with $l(\tilde{p}) \leq n$ and $l(c) \geq 1$. Now, we shall consider a path $\tilde{p}$. It is clear that $w(p) \leq w(\tilde{p})$ and hence we get

$$
x(l(\tilde{p})) \geq a_{i k}^{l(\tilde{p})} \otimes \underline{x}_{k} \geq w(\tilde{p}) \otimes \underline{x}_{k} \geq w(p) \otimes \underline{x}_{k}=a_{i k}^{n+1} \otimes \underline{x}_{k}>\bigoplus_{s \in N} a_{i s}^{n} \otimes \underline{x}_{s}=\underline{x}(n) .
$$

This is a contradiction with (3) and $(5)$ follows.

Corollary 5.4. Let $\boldsymbol{X}$ be invariant under $A$. Then $\{\underline{x}(n), \bar{x}(n)\} \subseteq V(A) \cap \boldsymbol{X}$.

Proof. The assertions follows from the facts that

$$
\begin{aligned}
& A \otimes \underline{x}(n)=A \otimes\left(A^{n} \otimes \underline{x}\right)=A^{n+1} \otimes \underline{x}=\underline{x}(n+1)=\underline{x}(n), \\
& A \otimes \bar{x}(n)=A \otimes\left(A^{n} \otimes \bar{x}\right)=A^{n+1} \otimes \bar{x}=\bar{x}(n+1)=\bar{x}(n) .
\end{aligned}
$$

Theorem 5.5. Let $\boldsymbol{X}$ be invariant under $A$. Then $x^{\oplus}(A, \boldsymbol{X})=\bar{x}(n)$. 
Pro of. Suppose that $\boldsymbol{X}$ is invariant under $A$ and $x^{\oplus}(A, \boldsymbol{X})=\left(x_{1}^{\oplus}(A, \boldsymbol{X}), \ldots, x_{n}^{\oplus}(A, \boldsymbol{X})\right)^{T}$. Then by the definition of $x_{k}^{\oplus}(A, \boldsymbol{X})$ there is a path $p=p^{\prime} \cup c$ ( $p$ is finished by $c$ ) in $\tilde{G}\left(A_{(h)}, \bar{x}_{(h)}\right)$ for $h=x_{k}^{\oplus}(A, \boldsymbol{X})$ and $w(p)=h$. Consider now a path $p^{\prime \prime}=p^{\prime} \cup c \cup \cdots \cup c$ with $l\left(p^{\prime \prime}\right) \geq n$ and $w(p)=w\left(p^{\prime \prime}\right)$. Then we obtain

$$
\bar{x}_{k}(n)=\bar{x}_{k}\left(l\left(p^{\prime \prime}\right)\right) \geq w\left(p^{\prime \prime}\right)=x_{k}^{\oplus}(A, \boldsymbol{X}) .
$$

Inverse inequality follows from the fact that a path $p \in \mathcal{P}_{k j}^{n}$ beginning in $k$ contains a cycle and $\bar{x}_{k}(n)=\bigoplus_{j} a_{k j}^{n} \otimes \bar{x}_{j}=a_{k \ell}^{n} \otimes \bar{x}_{\ell}=w(p) \otimes \bar{x}_{j}$, i.e., $k$ is precyclic in $\tilde{G}\left(A_{(h)}, \bar{x}_{(h)}\right)$ for $h=\bar{x}_{k}(n)$. Hence $x^{\oplus}(A, \boldsymbol{X}) \geq \bar{x}(n)$.

\subsection{Computing the greatest $\mathrm{X}$-eigenvector - general case}

In this subsection we shall analyze the conditions for computing $x^{\oplus}(A, \boldsymbol{X})$ whereby $\boldsymbol{X}$ is not invariant under $A$.

Let $A \in \mathbb{B}(n, n)$ and $\boldsymbol{X} \subseteq \mathbb{B}(n)$ be given. Suppose that $A \otimes \bar{x} \not \bar{x}$ and $V(A) \cap \boldsymbol{X} \neq \emptyset$, i.e., $x^{\oplus}(A, \boldsymbol{X})$ exists. We look for the greatest vector $\tilde{x} \in \boldsymbol{X}$ with the property that $\underline{x} \leq A \otimes \tilde{x} \leq \tilde{x}$. For this purpose $\tilde{x}$ will be constructed by the following algorithm.

\section{Algorithm Invariant upper bound}

Input. $\boldsymbol{X}, A$.

Output. "yes" in variable gr if $\tilde{x} \in \boldsymbol{X}$ with the property that $\underline{x} \leq A \otimes \tilde{x} \leq \tilde{x}$ exists; "no" in gr otherwise.

\section{begin}

1. $\tilde{x}:=\bar{x} ; M:=\emptyset ;$

2. $m:=\min _{j \in N \backslash M} \tilde{x}_{j} ; M=\left\{j \in N ; m=\tilde{x}_{j}\right\}$;

3. While $(\exists k \in M)(\exists j \in N)\left[a_{k j} \otimes \tilde{x}_{j}>\tilde{x}_{k}\right]$ do

$$
\text { if } \tilde{x}_{k} \geq \underline{x}_{j} \text { then } \tilde{x}_{j}:=m \wedge M:=M \cup\{j\} \text { else gr:="no"; }
$$

(comment: if the condition $A \otimes \tilde{x} \leq \tilde{x}$ is not fulfilled in row $k$, corresponding variables $\tilde{x}$ are modified)

4. $M=\left\{j \in N ; m \geq \tilde{x}_{j}\right\}$;

(comment: $M$ consists of row indices for which $A \otimes \tilde{x} \leq \tilde{x}$ holds true)

5. If $N \backslash M=\emptyset$ then gr:="yes" else go to 2 ;

\section{end}


Example 5.6. Let $A$ and $\boldsymbol{X}=[\underline{x}, \bar{x}]$ have the forms

$$
A=\left(\begin{array}{lllllll}
1 & 1 & 1 & 3 & 5 & 2 & 8 \\
2 & 7 & 4 & 3 & 2 & 5 & 6 \\
1 & 2 & 7 & 4 & 1 & 1 & 0 \\
2 & 2 & 5 & 4 & 2 & 6 & 4 \\
1 & 1 & 4 & 3 & 2 & 5 & 1 \\
2 & 3 & 5 & 2 & 1 & 2 & 2 \\
3 & 2 & 6 & 6 & 2 & 6 & 4
\end{array}\right), \quad \underline{x}=\left(\begin{array}{l}
3 \\
3 \\
3 \\
3 \\
3 \\
3 \\
3
\end{array}\right), \quad \bar{x}=\left(\begin{array}{l}
6 \\
7 \\
6 \\
5 \\
4 \\
4 \\
7
\end{array}\right)
$$

The first run of the algorithm:

By applying step 1 of the algorithm put $\tilde{x}:=\bar{x}, M:=\emptyset$ and

$$
m:=\min _{j \in N \backslash M} \tilde{x}_{j}=4, M=\left\{j \in N ; m=\tilde{x}_{j}\right\}=\{5,6\}
$$

in step 2. Since $a_{63} \otimes \tilde{x}_{3}>\tilde{x}_{6}$, put $\tilde{x}_{3}:=m=4\left(\geq \underline{x}_{3}\right), M=\left\{j \in N ; m=\tilde{x}_{j}\right\}=\{3,5,6\}$ and $\tilde{x}=(6,7,4,5,4,4,7)^{T}$. In step 4 the set $M=\left\{j \in N ; m \geq \tilde{x}_{j}\right\}=\{3,5,6\}$, in step 5 we obtain $N \backslash M \neq \emptyset$ and the algorithm goes on step 2 .

The second run of the algorithm:

In step 2 we get

$$
m:=\min _{j \in N \backslash M} \tilde{x}_{j}=5, M=\left\{j \in N ; m=\tilde{x}_{j}\right\}=\{4\}
$$

Since the condition of step 3 is not fulfilled $\left((A \otimes \tilde{x})_{4} \leq \tilde{x}_{4}\right)$, we continue by step 4 and step 5 , i.e., $M=\left\{j \in N ; m \geq \tilde{x}_{j}\right\}=\{3,4,5,6\}, N \backslash M \neq \emptyset$ and the algorithm goes on step 2 .

The third run of the algorithm:

In step 2 we get

$$
m:=\min _{j \in N \backslash M} \tilde{x}_{j}=6, M=\left\{j \in N ; m=\tilde{x}_{j}\right\}=\{1\}
$$

Step 3 produces the following $a_{17} \otimes \tilde{x}_{7}>\tilde{x}_{1}$, put $\tilde{x}_{7}:=m=6\left(\geq \underline{x}_{7}\right)$, and $\tilde{x}=$ $(6,7,4,5,4,4,6)^{T}$. Since $M=\left\{j \in N ; m \geq \tilde{x}_{j}\right\}=N \backslash\{2\}$, the algorithm again goes on step 2 .

The fourth run of the algorithm:

In step 2 we get

$$
m:=\min _{j \in N \backslash M} \tilde{x}_{j}=7, M=\left\{j \in N ; m=\tilde{x}_{j}\right\}=\{2\} .
$$

Since the condition of step 3 is not fulfilled and $M=N$ the algorithm terminates in step 5 with the variable gr:="yes" and output $\tilde{x}=(6,7,4,5,4,4,6)^{T}$. 
Theorem 5.7. Let $A \in \mathbb{B}(n, n)$ and $\boldsymbol{X} \subseteq \mathbb{B}(n)$ be given. Then the algorithm Invariant upper bound is correct, its output is the greatest vector $\tilde{x}$ such that $\underline{x} \leq A \otimes \tilde{x} \leq \tilde{x}$ and its computational complexity is $O\left(n^{2}\right)$.

Proof. The algorithm finishes with the positive answer in step 5, where it has computed the vector $\tilde{x}$ such that $\underline{x} \leq A \otimes \tilde{x} \leq \tilde{x}$.

If $\tilde{x}$ does not exists (this corresponds to else branch of the algorithm in step 3) then it is impossible to decrease $\tilde{x}_{j}$ on the level $\tilde{x}_{k}$ if $a_{k j} \otimes \tilde{x}_{j}>\tilde{x}_{k}$ and $\tilde{x}_{k}<\underline{x}_{j}$. Further, the smallest possible decrease of $\tilde{x}_{j}$ in step 3 guarantees the maximality of $\tilde{x} \in \boldsymbol{X}$ with property $\underline{x} \leq A \otimes \tilde{x} \leq \tilde{x}$.

For the estimation of the computational complexity observe that the algorithm checks for each coordinate $\tilde{x}_{k}$ at most $n$ products $a_{k j} \otimes \tilde{x}_{j}$ and compares it with $\tilde{x}_{k}$ in step 3 . The number of operations in step 3 is $O\left(n^{2}\right)$ and in no other step it exceeds the bound $O(n)$, hence the overall complexity is $O\left(n^{2}\right)$.

Now, we can summarize the above results and suggest an algorithm for computing the greatest $\boldsymbol{X}$-eigenvector of $A$.

\section{Algorithm Greatest X-eigenvector}

Input. $\boldsymbol{X}, A$.

Output. "yes" in variable gr if $x^{\oplus}(A, \boldsymbol{X}) \in \boldsymbol{X}$ exists; "no" in gr otherwise.

\section{begin}

1. If $A \otimes \bar{x} \not \leq \bar{x}$ then compute $\tilde{x}$ by the algorithm Invariant upper bound; put $\bar{x}:=\tilde{x}$

2. Compute $\bar{x}(n)=A^{n} \otimes \bar{x}$;

3. If $\underline{x} \leq A^{n} \otimes \bar{x}$ then $x^{\oplus}(A, \boldsymbol{X}):=\bar{x}(n)$ else $x^{\oplus}(A, \boldsymbol{X})$ does not exist;

end

Theorem 5.8. The algorithm Greatest X-eigenvector correctly computes $x^{\oplus}(A, \boldsymbol{X})$ in $O\left(n^{3}\right)$ arithmetic operations.

Proof. To determine a complexity of the algorithm, recall first that computing $\tilde{x}$ by the algorithm Invariant upper bound in step 1 needs $O\left(n^{2}\right)$ operations. The number of operations for computing $x(n)$ is $n O\left(n^{2}\right)=O\left(n^{3}\right)$. Thus, the complexity of the whole algorithm is $O\left(n^{3}\right)$.

\subsection{Applications of the greatest $\mathrm{X}$-eigenvector}

$\mathrm{n}$ this section we will analyze conditions for matrices under which multi-processor interaction systems reach the greatest steady state with any starting vector belonging to an interval vector $\boldsymbol{X}$. 
The set of starting vectors from which a multi-processor interaction system reaches an eigenvector (the greatest eigenvector) of $A$ after a finite number of stages, is called attraction set (strongly attraction set) of $A$, see [1, 19] ([14, 15]). In general, attraction set (strongly attraction set) contains the set of all eigenvectors, the set of all eigenvectors belonging to $\boldsymbol{X}$ but it can be also as big as the whole space. Let us denote the sets $\operatorname{attr}(A)$ and $\operatorname{attr}^{*}(A)$ as follows

$$
\begin{gathered}
\operatorname{attr}(A)=\{x \in \mathbb{B}(n) ; O(A, x) \cap V(A) \neq \emptyset\}, \\
\operatorname{attr}^{*}(A)=\left\{x \in \mathbb{B}(n) ; x^{*}(A) \in O(A, x)\right\} .
\end{gathered}
$$

The set $\operatorname{attr}(A)\left(\operatorname{attr}^{*}(A)\right)$ allows us to characterize matrices for which an eigenvector (the greatest eigenvector) is reached with any starting vector. It is easy to see that $x^{*}(A) \geq c^{*}(A)$ holds true and $x^{*}(A)$ can not be reached with a vector $x \in \mathbb{B}(n)$, $x<c^{*}(A)$.

Let us denote the set $\left\{x \in \mathbb{B}(n) ; x<c^{*}(A)\right\}$ by $M(A)$.

Definition 5.9. $A \in \mathbb{B}(n, n)$ is called strongly robust if $\operatorname{attr}^{*}(A)=\mathbb{B}(n) \backslash M(A)$.

Theorem 5.10. (Plavka and Szabó [14]) Let $A \in \mathbb{B}(n, n)$ be a matrix. Then $A$ is strongly robust if and only if $x^{*}(A)=c^{*}(A)$ and $G\left(A_{(c(A)}\right)$ is a strongly connected digraph with period equal to 1 .

The concepts of strong robustness has been studied in [14, equivalent conditions and efficient algorithms for interval cases have been presented in [15].

Now we will consider the interval version of the strongly attraction set.

Definition 5.11. Let $A, \boldsymbol{X}$ be given. Then the strongly attraction $\operatorname{set} \operatorname{attr}^{*}(A, \boldsymbol{X})$ is defined as follows

$$
\operatorname{attr}^{*}(A, \boldsymbol{X})=\left\{x \in \mathbb{B}(n) ; x^{*}(A, \boldsymbol{X}) \in O(A, x)\right\} .
$$

Definition 5.12. Let $A, \boldsymbol{X}$ be given. $A$ is called strongly $\boldsymbol{X}$-robust if $\boldsymbol{X} \subseteq \operatorname{attr}^{*}(A, \boldsymbol{X})$.

Lemma 5.13. If $A$ is strongly $\boldsymbol{X}$-robust then $(\forall x \in \boldsymbol{X})[\operatorname{per}(A, x)=1]$.

Pr o of. Suppose that $A$ is strongly $\boldsymbol{X}$-robust and $x \in \boldsymbol{X}$ is an arbitrary vector. Then there is $k \in \mathbb{N}$ such that $x(k)=x^{*}(A, \boldsymbol{X})$ and we obtain the following

$$
x(k)=x^{*}(A, \boldsymbol{X})=A \otimes x^{*}(A, \boldsymbol{X})=A \otimes x(k)=x(k+1)
$$

and the assertion follows.

Theorem 5.14. Let $A \in \mathbb{B}(n, n)$ and $\boldsymbol{X}$ be given. Then $A$ is strongly $\boldsymbol{X}$-robust if and only if $A^{n} \otimes \underline{x}=x^{*}(A, \boldsymbol{X})=A^{n} \otimes \bar{x}$. 
Proof. Suppose that $A \in \mathbb{B}(n, n)$ and $\boldsymbol{X}$ are given and $A^{n} \otimes \underline{x}=x^{*}(A, \boldsymbol{X})=A^{n} \otimes \bar{x}$. Then for an arbitrary vector $x \in \boldsymbol{X}$ we get (by monotonicity of $\otimes$ ) the following

$$
x^{*}(A, \boldsymbol{X})=A^{n} \otimes \underline{x} \leq A^{n} \otimes x \leq A^{n} \otimes \bar{x}=x^{*}(A, \boldsymbol{X}) .
$$

The converse implication is trivial by Lemma 5.2 and Lemma 5.3 .

Notice that according to the last theorem the complexity of a procedure for checking strong $\boldsymbol{X}$-robustness of a given matrix $A$ is $O\left(n^{3}\right)$ consisting of computing $x^{*}(A, \boldsymbol{X})$ in $O\left(n^{3}\right)$ steps and each of vectors $\underline{x}(1), \ldots, \underline{x}(n), \bar{x}(1), \ldots, \bar{x}(n)$ in $O\left(n^{2}\right)$ operations. Thus whole procedure has the computational complexity equal to $O\left(n^{3}\right)+2 n O\left(n^{2}\right)=O\left(n^{3}\right)$.

\section{ACKNOWLEDGMENTS}

The author would like to thank the referees for their valuable remarks and suggestions that helped to increase the clarity of arguments in the paper.

This work was supported by grants GAČR \#14 - 02424S and APVV-04-04-12.

(Received January 27, 2015)

\section{REFERENCES}

[1] P. Butkovič: Max-linear Systems: Theory and Applications. Springer, 2010. DOI:10.1007/978-1-84996-299-5

[2] K. Cechlárová: Eigenvectors in Bottleneck algebra. Linear Algebra Appl. 175 (1992), 63-73. DOI:10.1016/0024-3795(92)90302-q

[3] M. Fiedler, J. Nedoma, J. Ramík, J. Rohn, and K. Zimmermann: Linear Optimization Problems with Inexact Data. Springer-Verlag, Berlin 2006.

[4] M. Gavalec and K. Zimmermann: Classification of solutions to systems of two-sided equations with interval coefficients. Int. J. Pure Appl. Math. 45 (2008), 533-542.

[5] M. Gavalec: Periodicity in Extremal Algebra. Gaudeamus, Hradec Králové 2004.

[6] M. Gavalec and J. Plavka: Fast algorithm for extremal biparametric eigenproblem. Acta Electrotechnica et Informatica 7 (2007), 3.

[7] M. Gavalec and J. Plavka: Monotone interval eigenproblem in max-min algebra. Kybernetika 46 (2010), 3, 387-396.

[8] V. Lacko and Š. Berežný: The color-balanced spanning tree problem. Kybernetika 41 (2005), 539-546.

[9] M. Molnárová and J. Pribiš: Matrix period in max-algebra. Discrete Applied Mathematics 103 (2000), 167-175. DOI:10.1016/s0166-218x(99)00242-5

[10] M. Molnárová, H. Myšková, and J. Plavka: The robustness of interval fuzzy matrices. Linear Algebra and Its Appl. 438 (2013), 8, 3350-3364. DOI:10.1016/j.laa.2012.12.020

[11] H. Myšková: Weak stability of interval orbits of circulant matrices in fuzzy algebra. Acta Electrotechnica et Informatica 12 (2012), 3, 51-56. DOI:10.2478/v10198-012-0032-4

[12] H. Myšková: Interval eigenvectors of circulant matrices in fuzzy algebra. Acta Electrotechnica et Informatica 12 (2012), 3, 57-61. DOI:10.2478/v10198-012-0033-3 
[13] J. Plavka and P. Szabó: The $O\left(n^{2}\right)$ algorithm for the eigenproblem of an $\epsilon$-triangular Toeplitz matrices in max-plus algebra. Acta Electrotechnica et Informatica 9 (2009), 4, $50-54$.

[14] J. Plavka and P. Szabó: On the $\lambda$-robustness of matrices over fuzzy algebra. Discrete Applied Math. 159 (2011), 5, 381-388. DOI:10.1016/j.dam.2010.11.020

[15] J. Plavka: On the $O\left(n^{3}\right)$ algorithm for checking the strong robustness of interval fuzzy matrices. Discrete Applied Math. 160 (2012), 640-647. DOI:10.1016/j.dam.2011.11.010

[16] J. Plavka: On the weak robustness of fuzzy matrices. Kybernetika 49 (2013), 1, 128-140.

[17] J. Rohn: Systems of linear interval equations. Linear Algebra and Its Appl. 126 (1989), 39-78. DOI:10.1016/0024-3795(89)90004-9

[18] E. Sanchez: Resolution of eigen fuzzy sets equations. Fuzzy Sets and Systems 1 (1978), 69-74. DOI:10.1016/0165-0114(78)90033-7

[19] S. Sergeev: Max-algebraic attraction cones of nonnegative irreducible matrices. Linear Algebra Appl. 435 (2011), 7, 1736-1757. DOI:10.1016/j.laa.2011.02.038

[20] Yi-Jia Tan: Eigenvalues and eigenvectors for matrices over distributive lattices. Lin. Algebra Appl. 283 (1998), 257-272. DOI:10.1016/s0024-3795(98)10105-2

[21] K. Zimmermann: Extremální algebra (in Czech). Ekon. ústav ČSAV Praha, 1976.

Ján Plavka, Department of Mathematics and Theoretical Informatics, Faculty of Electrical Engineering and Informatics, Technical University in Košice, B. Němcovej 32, 04200 Košice. Slovak Republic.

e-mail: Jan.Plavka@tuke.sk 